\title{
EULER CHARACTERISTIC NUMBERS OF SPACE-LIKE MANIFOLDS
}

\author{
BING-LONG CHEN, KUN ZHANG
}

\begin{abstract}
In this note, we prove that if a compact even dimensional manifold $M^{n}$ with negative sectional curvature is homotopic to some compact space-like manifold $N^{n}$, then the Euler characteristic number of $M^{n}$ satisfies $(-1)^{\frac{n}{2}} \chi\left(M^{n}\right)>0$. We also show that the minimal volume conjecture of Gromov is true for all compact even dimensional space-like manifolds.
\end{abstract}

\section{INTRODUCTION}

Let $M^{n}$ be a compact even dimensional Riemannian manifold with negative sectional curvature. A long-standing conjecture due to H. Hopf 5 in differential geometry asks whether the Euler characteristic number of $M^{n}$ satisfies $(-1)^{\frac{n}{2}} \chi\left(M^{n}\right)>0$. When $n=4$, the proof was given by Chern[2] (who attributed the result to Milnor) by showing the integrand of Gauss-Bonnet-Chern is positive. However, when $n=6$, some examples show that the integrand does not have a definite sign in general. On the other hand, Gromov in 4] proved that Hopf conjecture is true when the manifold is Kähler.

In this note, we will consider the Euler characteristic numbers of a class of real Riemannian manifolds. These manifolds are locally embeddable in LorentzMinkowski space $R^{n, 1}$. In [7], such $N^{n}$ is called space-like. More precisely, we call a manifold $\left(N^{n}, g\right)$ (see [7]) space-like if there exists a symmetric $(0,2)$ tensor $h_{i j}$ such that the following two equations are fulfilled

$$
\begin{gathered}
R_{i j k l}=-\left(h_{i k} h_{j l}-h_{i l} h_{j k}\right) \\
\nabla_{i} h_{j k}=\nabla_{j} h_{i k} .
\end{gathered}
$$

Here the sign convention for the Riemann curvature tensor $R_{i j k l}$ is made so that $R_{i j i j}$ is positive on sphere. Clearly, a space-like $n$-dimensional submanifold of $R^{n, 1}$ satisfies the above two equations (1.1) and (1.2) if we take the tensor $h_{i j}$ to be the second fundamental form. A space-like manifold shares some interesting properties of manifolds with non-positive curvature. For example, it can be shown that the universal cover of a space-like manifold is diffeomorphic to the Euclidean space (see Corollary 2.2). The main result of this note is the following theorem:

Theorem 1.1. Let $M^{n}$ be an even dimensional Riemannian manifolds with negative sectional curvature. Suppose $M^{n}$ is homotopic to some compact space-like manifold $N^{n}$. Then the Euler characteristic number of $M^{n}$ satisfies

$$
(-1)^{\frac{n}{2}} \chi\left(M^{n}\right)>0 .
$$

Note that in the theorem, we do not assume the curvature of space-like manifold $N^{n}$ has a sign. The curvature sign is only imposed on the manifold $M^{n}$. The 
motivation for the proof of Theorem 1.1 is from [7, where the second author studied the hyperbolization problem of space-like manifolds by using the so-called intrinsic mean curvature flow. More precisely, it was shown in [7 that if the manifold is compact and $h_{i j}>0$, then the manifold admits a Riemannian metric of negative constant sectional curvature.

Theorem 1.1 follows from a more general result on space-like manifolds satisfying (1.1) and (1.2):

Theorem 1.2. Let $N^{n}$ be an even dimensional compact space-like manifold, then the Euler characteristic number satisfies

$$
(-1)^{\frac{n}{2}} \chi\left(M^{n}\right)>0 .
$$

The equality holds if and only if the minimal volume of $N^{n}$ is zero.

According to Gromov [3], the minimal volume of a manifold $N^{n}$ is the infimum of all volumes $\operatorname{vol}\left(N^{n}, g^{\prime}\right)$, where $g^{\prime}$ ranges over all Riemannian metrics with sectional curvatures satisfying $\left|K_{g^{\prime}}\right| \leq 1$. The minimal volume conjecture of Gromov [3] is asking whether there is a number $\varepsilon(n)$ depending only on the dimension $n$ such that $\min \operatorname{vol}\left(N^{n}\right)<\varepsilon(n)$ implies min vol $\left(N^{n}\right)=0$. This conjecture was already verified by X. C. Rong [6] in dimension 4. A byproduct of Theorem 1.2 is

Corollary 1.3. The minimal volume conjecture is true for all compact even dimensional space-like manifolds.

The proof of above Theorems 1.1 and 1.2 is an elementary application of the mean curvature flow. The detail will be given in the following two sections.

\section{Mean Curvature Flow}

In this section, we assume that $\left(N^{n}, g\right)$ is a space-like manifold, i.e. there is a tensor $h_{i j}$ such that equation (1.1) and (1.2) hold. In 7], the second author studied the following flow:

$$
\begin{aligned}
\frac{\partial g_{i j}}{\partial t}= & -2 R_{i j}+2 h_{i k} h_{j l} g^{k l}, \\
\frac{\partial h_{i j}}{\partial t}= & \triangle h_{i j}-R_{i m} h_{n j} g^{m n}-R_{j m} h_{n i} g^{m n} \\
& +2 h_{i m} h_{j n} h_{k l} g^{m k} g^{l n}-h_{m n} h_{k l} g^{m k} g^{n l} h_{i j} .
\end{aligned}
$$

It was shown in [7, when $\left(N^{n}, g\right)$ is compact, equation (2.1) admits a smooth solution for any initial data $\left(g_{0}, h_{0}\right)$. Moreover, if equations (1.1) and (1.2) hold at time $t=0$, then they also continue to hold for time $t>0$. That is to say, $\left(N^{n}, g(t), h(t)\right)$ will remain to be a space-like manifold under the deformation (2.1). In this case, equation (2.1) may be simplified:

$$
\begin{aligned}
\frac{\partial g_{i j}}{\partial t} & =2 H h_{i j} \\
\frac{\partial h_{i j}}{\partial t} & =\triangle h_{i j}+2 H h_{i m} h_{j n} g^{m n}-|A|^{2} h_{i j}
\end{aligned}
$$

where $H=g^{i j} h_{i j},|A|^{2}=g^{i j} g^{k l} h_{i k} h_{j l}$.

We may call equation (2.1) an intrinsic mean curvature flow. Equations (1.1) and (1.2) may be called Gauss and Codazzi equations respectively. 
Another approach to solve equation (2.1) or (2.2) is to embed the universal cover $(\tilde{N}, \tilde{g})$ into $R^{n, 1}$ as a space-like submanifold $\Sigma$ in the usual sense, deform $\Sigma$ in $R^{n, 1}$ by the mean curvature flow and prove the mean curvature flow is invariant under the deck transformation.

Proposition 2.1. $\tilde{N}$ admits an isometric embedding into $R^{n, 1}$ as a space-like submanifold with second fundamental form $h_{i j}$ given in (1.1) and (1.2).

Proof. By a monodromy argument, there is a smooth isometric immersion $\varphi$ : $(\tilde{N}, \tilde{g}) \rightarrow R^{n, 1}$ with $h_{i j}$ as the second fundamental form. Let $\pi: R^{n, 1} \rightarrow R^{n}$ be the projection to an $n$-coordinate plane, and $\psi=\pi \circ \varphi$. Let $g_{0}$ be the Euclidean metric in $R^{n}$, then it is not hard to see $\tilde{g} \leq \psi^{*} g_{0}$. This implies $\psi$ is proper, hence a covering map to $R^{n}$. From this, we know $\varphi$ is an embedding.

Corollary 2.2. The universal cover $\tilde{N}$ is diffeomorphic to the Euclidean space.

Now let $\left(N^{n}, g\right)$ be a compact space-like manifold, we deform $(g, h)$ by (2.1) or (2.2).

From (2.2), it is not hard to show

$$
\begin{aligned}
\frac{\partial H}{\partial t} & =\triangle H-H|A|^{2} \\
\frac{\partial|A|^{2}}{\partial t} & =\triangle|A|^{2}-2|\nabla A|^{2}-2|A|^{4}
\end{aligned}
$$

where $|\nabla A|^{2}=g^{i j} g^{k l} g^{p q} \nabla_{i} h_{k p} \nabla_{j} h_{l q}$.

Based on (2.3), it can be shown that the solution $(g(t), h(t))$ of equation (2.1) (or (2.2)) always exists for all time $0 \leq t<\infty$, and $h_{i j}$ satisfies the estimate

$$
0 \leq|A|^{2} \leq \frac{1}{2 t+1 /|A|_{\max }(0)}
$$

In the following propositions, we assume the dimension $n$ to be even.

First, we derive two monotonicity formulas for the intrinsic mean curvature flow. We have to mention that all the quantities in the following propositions involving the norm and the volume element $d v$ are computed at time $t$ with the evolving metric $g(t)$.

\section{Proposition 2.3.}

$$
\begin{aligned}
& \frac{d}{d t} \int_{N^{n}} H^{n} d v=-n(n-1) \int_{N^{n}}|\nabla H|^{2} H^{n-2} d v-n \int_{N^{n}} H^{n}\left|h_{i j}-\frac{H}{n} g_{i j}\right|^{2} d v \leq 0 . \\
& \text { and } \\
& \qquad \begin{aligned}
\frac{d}{d t} \int_{N^{n}}|A|^{n} d v= & -\left.\left.\left(\frac{n}{2}-1\right) \frac{n}{2} \int_{N^{n}}|A|^{(n-4)}|\nabla| A\right|^{2}\right|^{2} d v-n \int_{N^{n}}|A|^{n-2}|\nabla A|^{2} d v \\
& -n \int_{N^{n}}|A|^{n}\left|h_{i j}-\frac{H}{n} g_{i j}\right|^{2} d v \leq 0 .
\end{aligned}
\end{aligned}
$$

Proof. The proof is direct calculations by using equations (2.3).

Proposition 2.4. There are constant $C_{0}>0$ and a sequence of times $t_{k} \rightarrow \infty$ such that

$$
0 \leq \int_{N^{n}}|A|^{n} d v<C_{0}
$$


for all time $t \geq 0$, and

$$
\left.t_{k} \cdot \int_{N^{n}}|A|^{n}\left|h_{i j}-\frac{H}{n} g_{i j}\right|^{2} d v\right|_{t=t_{k}} \rightarrow 0 \quad \text { as } \quad k \rightarrow \infty .
$$

Proof. Integrating the time in the first formula of Proposition 2.3 from $-\infty$ to 0 , we have

$$
\int_{-\infty}^{0} \int_{N^{n}}|A|^{n}\left|h_{i j}-\frac{H}{n} g_{i j}\right|^{2} d V \leq C_{0} .
$$

If (2.6) does not hold, then there is $C>0$ such that for all $t>0$, we have

$$
\int_{N^{n}}|A|^{n}\left|h_{i j}-\frac{H}{n} g_{i j}\right|^{2} d v>\frac{1}{t+C},
$$

which is a contradiction with (2.7).

Since

$$
\frac{d}{d t} \operatorname{Vol}\left(N^{n}, t\right)=\int_{N^{n}} H^{2} d v \leq\left(\int_{N^{n}}|H|^{n} d v\right)^{\frac{2}{n}}\left(\operatorname{Vol}\left(N^{n}, t\right)\right)^{1-\frac{2}{n}},
$$

we have

$$
\frac{d}{d t} \operatorname{Vol}\left(N^{n}, t\right)^{\frac{2}{n}} \leq \frac{2}{n}\left(\int_{N^{n}}|H|^{n} d v\right)^{\frac{2}{n}}
$$

This implies

Proposition 2.5. There is a constant $C_{1}>0$ such that for all $t>0$

$$
\begin{aligned}
& \operatorname{Vol}\left(N^{n}, t\right) \leq C_{1}(t+1)^{\frac{n}{2}}, \\
& \frac{1}{1+t} \int_{N^{n}}|A|^{n-2} d V \leq C_{1} .
\end{aligned}
$$

Moreover when $n$ is even

$$
\limsup _{t \rightarrow \infty} \frac{\operatorname{Vol}\left(N^{n}, t\right)}{(1+t)^{\frac{n}{2}}} \leq\left(\frac{2}{n}\right)^{\frac{n}{2}} \lim _{t \rightarrow \infty} \int_{N^{n}}|H|^{n} d v .
$$

Proof. From (2.8) we have

$$
\frac{\operatorname{Vol}\left(N^{n}, t\right)^{\frac{2}{n}}-\operatorname{Vol}\left(N^{n}, 0\right)^{\frac{2}{n}}}{t+1} \leq \frac{2}{n} \frac{\int_{0}^{t}\left(\int_{N^{n}}|H|^{n} d v\right)^{\frac{2}{n}} d t}{t+1} .
$$

Since $n$ is even, and $\int_{N^{n}}|H|^{n} d v$ is monotonically decreasing from the first formula in Proposition 2.1, we know

$$
\limsup _{t \rightarrow \infty} \frac{\operatorname{Vol}\left(N^{n}, t\right)^{\frac{2}{n}}}{1+t} \leq \frac{2}{n} \lim _{t \rightarrow \infty}\left(\int_{N^{n}}|H|^{n} d v\right)^{\frac{2}{n}} .
$$




\section{Proof of the Main theorem}

To prove Theorem 1.2, we may assume $N^{n}$ is orientable. It is well-known that by Gauss-Bonnet-Chern theorem, the Euler Characteristic number $\chi\left(N^{n}\right)$ may be expressed as a curvature integral (see Chern [1]):

$$
\int_{N^{n}} \Omega=\chi\left(N^{n}\right)
$$

where

$$
\Omega=\frac{1}{2^{n} \pi^{\frac{n}{2}}\left(\frac{n}{2}\right) !} \sum \varepsilon^{i_{1}, i_{2}, \cdots, i_{n}} \Omega_{i_{1} i_{2}} \wedge \cdots \Omega_{i_{n-1} i_{n}}
$$

and $\Omega_{i_{1} i_{2}}=R_{i_{1} i_{2} j_{1} j_{2}} d x^{j_{1}} \wedge d x^{j_{2}}$ is the curvature form. From equation (1.1) and direct calculations, it follows

$$
\Omega=(-1)^{\frac{n}{2}} \frac{\Gamma\left(\frac{n+1}{2}\right)}{\pi^{\frac{n+1}{2}}} \frac{\operatorname{det}(h)}{\operatorname{det}(g)} d v=(-1)^{\frac{n}{2}} \frac{2}{\operatorname{vol}\left(S^{n}\right)} \frac{\operatorname{det}(h)}{\operatorname{det}(g)} d v
$$

and

$$
\chi\left(N^{n}\right)=(-1)^{\frac{n}{2}} \frac{2}{\operatorname{vol}\left(S^{n}\right)} \int_{N^{n}} \frac{\operatorname{det}(h)}{\operatorname{det}(g)} d v .
$$

We remark that equation (3.3) holds for any $t>0$, since equation (1.1) holds for any time $t>0$.

For any fixed $p \in N^{n}$, choose an orthonormal frame $e_{i}, i=1,2, \cdots, n$ such that $h_{i j}$ is diagonalied in this frame, i.e. $h_{i j}=\lambda_{i} \delta_{i j}$. Then we have

$$
\left|\frac{\operatorname{det}(h)}{\operatorname{det}(g)}-\left(\frac{H}{n}\right)^{n}\right| \leq\left|\lambda_{1} \cdots \lambda_{n}-\left(\frac{H}{n}\right)^{n}\right| \leq n|A|^{n-1}\left|h_{i j}-\frac{H}{n} g_{i j}\right| .
$$

Hence

$$
\begin{aligned}
& \int_{N^{n}}\left|\frac{\operatorname{det}(h)}{\operatorname{det}(g)}-\left(\frac{H}{n}\right)^{n}\right| d v \\
& \leq n \int_{N^{n}}|A|^{n-1}\left|h_{i j}-\frac{H}{n} g_{i j}\right| d v \\
& \leq n\left(\int_{N^{n}}|A|^{n}\left|h_{i j}-\frac{H}{n} g_{i j}\right|^{2} d v\right)^{\frac{1}{2}} \cdot\left(\int_{N^{n}}|A|^{n-2} d v\right)^{\frac{1}{2}} .
\end{aligned}
$$

Let $t_{k}$ be the time sequence chosen in Proposition 2.4, it follows from (2.6) and (2.10) that at $t=t_{k}$ :

$$
\left.\int_{N^{n}}|A|^{n}\left|h_{i j}-\frac{H}{n} g_{i j}\right|^{2} d v \cdot \int_{N^{n}}|A|^{n-2} d v\right|_{t=t_{k}} \rightarrow 0
$$

as $k \rightarrow \infty$. This implies

$$
\left.\lim _{k \rightarrow \infty} \int_{N^{n}}\left|\frac{\operatorname{det}(h)}{\operatorname{det}(g)}-\left(\frac{H}{n}\right)^{n}\right| d v\right|_{t=t_{k}}=0 .
$$

Combining (3.3) and (3.5), we have

$$
(-1)^{\frac{n}{2}} \chi\left(N^{n}\right)=\left.\lim _{k \rightarrow \infty} \frac{2}{\operatorname{vol}\left(S^{n}\right)} \int_{N^{n}}\left(\frac{H}{n}\right)^{n} d v\right|_{t=t_{k}} .
$$

Because $n$ is even, we know $(-1)^{\frac{n}{2}} \chi\left(N^{n}\right) \geq 0$. This finishes the main part of Theorem 1.2 . 
Clearly, if the minimal volume of $N^{n}$ is zero, then the Euler characteristic number $\chi\left(N^{n}\right)$ is zero. This follows directly from the Gauss-Bonnet-Chern formula (3.1). To see the converse, let $\chi\left(N^{n}\right)=0$, from (3.6) we have

$$
\left.\lim _{k \rightarrow \infty} \int_{N^{n}} H^{n} d v\right|_{t=t_{k}}=0 .
$$

Combining (2.11), it implies

$$
\limsup _{k \rightarrow \infty} \frac{\operatorname{Vol}\left(M, t_{k}\right)}{\left(1+t_{k}\right)^{\frac{n}{2}}}=0 .
$$

Note that (2.4) and (1.1) implies $|R m|\left(g_{t_{k}}\right) \leq C t_{k}^{-1}$. So $\frac{C g_{t_{k}}}{t_{k}}$ is a sequence of metrics with sectional curvatures satisfying $\left|K_{C g_{t_{k}}}\right| \leq 1$ but the volumes converges to zero as $k \rightarrow \infty$. This shows the minimal volume of $N^{n}$ is zero. The proof of Theorem 1.2 is completed.

To prove Theorem 1.1, we recall a result of Gromov [3]: the simplicial volume of a compact manifold $X^{n}$ with negative sectional curvature is positive. In our case, we have the simplicial volume of $M^{n}$ is positive, so is $N^{n}$ by the homotopic invariance of simplicial volume. In paper 3], Gromov proved that the minimal volume is always bounded from below by the simplicial volume multiplied by a constant depending only on the dimension. Theorem 1.1 follows from this result and Theorem 1.2. Finally, we mention one corollary:

Corollary 3.1. Let $\left(M^{n}, g, h\right)$ be an even-dimensional compact space-like manifold. Then

$$
\frac{1}{\operatorname{vol}\left(S^{n}\right)} \int_{M^{n}}|H|^{n} \geq(-1)^{\frac{n}{2}} \frac{n^{n}}{2} \chi\left(M^{n}\right) .
$$

Equality holds if and only if either $\left(M^{n}, g, h\right)$ is hyperbolic or flat.

Remark 3.2. It is desirable to generalize Theorem 1.2 to higher codimensional case. Namely, we may consider the manifold $\left(M^{n}, g\right)$ which is locally embeddable as a space-like higher codimensional submanifold of $R^{n, m}$. In this case, a formula similar to the first one in Proposition 2.3 can still hold:

$$
\frac{d}{d t} \int_{M^{n}}|H|_{+}^{n} \leq 0
$$

where $|H|_{+}^{n}$ is the absolute value of the squared norm of the (time-like) mean curvature vector. This in particular implies

$$
\limsup _{t \rightarrow \infty} \frac{\operatorname{Vol}\left(M^{n}, t\right)^{\frac{2}{n}}}{1+t} \leq \frac{2}{n} \lim _{t \rightarrow \infty}\left(\int_{M^{n}}|H|_{+}^{n} d v\right)^{\frac{2}{n}}<\infty .
$$

Acknowledgements The first author is partially supported by NSFC 11025107 , the second author by NSFC 11301190.

\section{REFERENCES}

[1] S.S. Chern, A simple intrinsic proof of the Gauss-Bonnet formula for closed Riemannian manifolds, Ann. of Math.(2) 45 (1944), 747-752.

[2] S.S. Chern, On curvature and characteristic classes of a Riemann manifold, Abn. Math. Sem. Univ. Hamburg 20 (1955), 117-126. 
[3] M. Gromov, Volume and bounded cohomology, Inst. Hautes. Études Sci. Publ. Math (1982), no. 56, 5-99.

[4] M. Gromov, Kähler hyperbolicity and $L^{2}$-Hodge theory, J. Differ. Geom. 33 (1991), 263-292.

[5] H. Hopf, Über die Curvatura integra geschlossener Hyperflächen, Math. Ann. 95 (1925), 340-367.

[6] X.C. Rong, The existence of polarized F-structures on volume collapsed 4-manifolds, Geom. Funct. Anal. 3 (1993), no. 5, 474-501.

[7] K. Zhang, Hyperbolic structures on closed space-like manifolds, Pac. J. Math. 243 (2009), no.1, 151-164. 